\title{
Selective Recognition of Bifunctional Molecules by Synthetic Polymers Prepared by Covalent Molecular Imprinting
}

\author{
Larysa Dubey ${ }^{1}$, Iva Chianella ${ }^{2}$, Igor Dubey ${ }^{1}$, Elena Piletska ${ }^{2}$, Michael J. Whitcombe ${ }^{2}$ and \\ Sergey Piletsky ${ }^{*}$, \\ ${ }^{1}$ Institute of Molecular Biology and Genetics, National Academy of Sciences, 150 Zabolotny str., 03143 Kyiv, Ukraine \\ ${ }^{2}$ Cranfield Health, Cranfield University, Bedfordshire, MK43 OAL, UK
}

\begin{abstract}
Affinity polymeric adsorbents useful for the separation of compounds which are derivatives of phenols, heterocycles and other compounds containing two polar moieties were prepared by a covalent imprinting approach. Such separation, for example, would facilitate the analytical detection and quantification of each individual compound especially when present in complex sample matrix. The synthetic method, utilised for the preparation of the polymers, is based on co-polymerization of diacrylate esters of hydroxyphenols (catechol, resorcinol and hydroquinone) with an appropriate cross-linker followed by alkaline hydrolysis of the esters and release of the corresponding phenol. The resulting swellable materials contain cavities with adjustable size and orientation of two carboxylic groups suited for the binding of molecules with appropriate size and complementary orientation of polar groups. In contrast to conventional MIPs the synthesized polymers have selectivity not only for the phenolic templates, but also for a group of compounds with suitable orientations of polar functionalities, such as aromatic nitrogen, amino or hydroxyl groups. Polymers demonstrated different affinity to ortho-, meta- and para-isomers of hydroxyphenols, hydroxypyridines and diazine heterocycles.
\end{abstract}

Keywords: Molecularly imprinted polymers, covalent imprinting, selectivity.

\section{INTRODUCTION}

During recent decades, the molecular imprinting approach [1] has been used in a variety of forms and applications [2-5]. In this technique a highly cross-linked polymer is formed around a template molecule. The template is then removed by washing and a cavity with functional groups complementary to those of the template molecule remains behind in the polymer [6]. Usually the polymers possess a high level of cross-linking to ensure fidelity of the binding sites for the target template. These polymers demonstrate very good thermal and mechanical stability and can be used in aggressive media [7]. The disadvantage of this approach for some industrial applications lies, surprisingly, in the unique selectivity of imprinted materials, which in most cases bind predominantly template molecules used in their preparation. Modern separation technology would sometimes benefit from the availability of generic adsorbents which can recognize not only the individual molecules used as templates (however the need for such materials also exists) but rather groups of compounds with similar structure.

In theory it would be possible to divide all molecules into several different groups which have common (or similar) orientations of 2-3 polar functional groups (determinants). Ideally 20-30 adsorbents capable of recognizing these determinants should be sufficient to resolve most of the

*Address correspondence to this author at the Cranfield Health, Cranfield University, Bedfordshire, MK43 0AL, UK; Tel: +44 1234 758323; Fax: +44 1234 758380; E-mail: s.piletsky@cranfield.ac.uk separation tasks existing in analytical science and in industry. The present work describes the initial steps in the development of polymeric adsorbents with two carboxylic groups, fixed inside binding cavities at a variable distance. These materials are capable of selective binding to molecules having two polar moieties such as e.g. hydroxy, amino groups or aromatic nitrogens that can interact with $-\mathrm{CO}_{2} \mathrm{H}$ groups. The synthetic approach used for the introduction of carboxylic acid groups into polymers was developed based on the covalent imprinting of polymerisable esters of bifunctional phenols (catechol, resorcinol and hydroquinone) with two acylated hydroxy groups in the ortho-, meta- and para-orientation, respectively. Co-polymerization of these diacryloyl derivatives provides polymers where ester bonds can be subsequently hydrolyzed with release of the corresponding phenol and the formation of binding cavities containing two carboxylic groups at different distances.

MIPs containing 4-chloro- and 4-nitrophenol have been previously prepared by covalent imprinting involving polymerization of the corresponding phenol acrylates, and nitro-(chloro-)phenyl methacrylates. The synthesized polymers were easily hydrolyzed to form the carboxylated polymer derivatives [8-10]. These polymers showed an imprint effect and could be employed e.g. for solid-phase extraction of the corresponding phenols from water. A specific recognition material for bisphenol A $\left(4,4^{\prime}\right.$ isopropylidenediphenol, BPA) was obtained by covalent imprinting using bisphenol A dimethacrylate as the template. When BPA was cleaved from the resulting polymer by alkaline hydrolysis of the ester bonds, carboxylic acid residues were generated in the polymer. The polymer 
adsorbed BPA and some structurally related compounds bearing two hydroxyl groups at the 4,4'-positions [11,12]. This type of covalent imprinting allows one to obtain polymeric adsorbents with an adjustable orientation of certain active groups, in this case carboxylic acid residues, suitable for the specific recognition of molecules with an appropriate size and complementary orientation of polar groups, able to interact with the polymeric binding sites. The "interval immobilization technique" of Kubo et al. [13,14] used a surrogate template to position ionisable functionality at a fixed distance to interact successfully with a target toxin. The strategy used in this paper has similarities to both of the above approaches, in that a covalent template has been used and fixed distances between functional groups in the polymer have been achieved. Rather than prepare materials for binding a specific target, the approach we have employed results in the synthesis of materials capable of selectively binding a range of molecules bearing two polar moieties, (such as amino or imino groups) dependent on the spacings of groups in the target matching those of complementary functionality in the polymer. Such materials could be used for the separation of polar compounds having very similar structure, facilitating their analytical detection and quantification.

\section{MATERIALS AND METHODOLOGY}

\section{Materials}

Catechol, resorcinol, hydroquinone, pyridazine, pyrimidine, pyrazine, 2-, 3- and 4-hydroxypyridine, triethylamine were obtained from Acros (UK). Acryloyl chloride, acrylic acid (AA), itaconic acid (IA), divinylbenzene, and 1,1'azobis(cyclohexanecarbonitrile) were from Aldrich (UK). Dimethylformamide (DMF) was obtained from BDH (UK), other solvents were purchased from Acros (UK).

\section{Synthesis of Monomers}

The phenol (catechol, resorcinol or hydroquinone), $4.4 \mathrm{~g}$ (40 mmol) and $14 \mathrm{~mL}$ of triethylamine $(100 \mathrm{mmol})$ in $75 \mathrm{~mL}$ of dry acetonitrile were cooled in an ice bath. A solution of acryloyl chloride $(8 \mathrm{~mL}, 100 \mathrm{mmol})$ in $25 \mathrm{~mL} \mathrm{CH}_{3} \mathrm{CN}$ was added dropwise with stirring over $30 \mathrm{~min}$. The stirring was continued for another 2 hours. The precipitate (triethylamine hydrochloride) was filtered off, washed with acetonitrile $(2 \times$ $20 \mathrm{~mL}$ ), and the solvent from the combined organic fractions was removed under reduced pressure. Chloroform $(200 \mathrm{~mL})$ was added to the residue; the resultant solution was washed with saturated aqueous $\mathrm{NaHCO}_{3}(2 \times 100 \mathrm{~mL})$, dried with anhydrous sodium sulfate, filtered and evaporated. The mixture was chromatographed on silica gel using chloroform as eluent. The product was then re-purified on a silica column eluting with $20 \%$ hexane in chloroform. Fractions containing the product were combined, evaporated and dried under vacuum.

Catechol diacrylate: yield $5.7 \mathrm{~g},(66 \%)$ was obtained as a colourless liquid that solidified upon storage at $4{ }^{\circ} \mathrm{C}$, forming a crystalline mass. Resorcinol diacrylate: yield 5.6 g (64\%), colourless liquid. Hydroquinone diacrylate: yield $5.3 \mathrm{~g}$ $(61 \%)$, white solid. No attempts were made to re-crystallize catechol and hydroquinone diacrylates. The structures of monomers were confirmed by mass-spectroscopy (ESI-MS) using Waters 2795 Separation Module in tandem with a bench-top triple quadrupole Micromass Quatro Micro mass spectrometer (Waters, UK) equipped with electrospray probe. All diacrylates produced identical mass spectra.

\section{Preparation of Polymers}

For the synthesis of imprinted polymers P1-P3, $300 \mathrm{mg}$ of the respective diacrylate monomer, $2.7 \mathrm{~g}$ of the crosslinker (divinylbenzene) and $30 \mathrm{mg}$ of initiator, 1,1'azobis(cyclohexanecarbonitrile), were dissolved in $3 \mathrm{~g}$ of dimethylformamide in a borosilicate glass tube. The reaction mixtures were de-aerated by passing nitrogen gas for $2 \mathrm{~min}$, then the tubes were tightly sealed and kept in a thermostatic oil bath at $80^{\circ} \mathrm{C}$ overnight $(18 \mathrm{~h})$. The bulk polymers were manually ground in methanol and mechanically wet-sieved through 106 and $45 \mu \mathrm{m}$ sieves (Endecotts, UK). Polymer particles with a size range of 45-106 $\mu \mathrm{m}$ were collected and dried.

Phenolic residues were cleaved from the polymers by treatment with $0.75 \mathrm{M} \mathrm{NaOH}$ in water-ethanol $3: 1$ for $10 \mathrm{~h}$ at $60{ }^{\circ} \mathrm{C}$ with occasional agitation. During this period, the alkaline solution was changed several times (polymer was filtered off, washed with $0.75 \mathrm{M} \mathrm{NaOH}$ and a fresh portion of $\mathrm{NaOH}$ solution was added). The hydrolyzed polymers were filtered off, washed with $50 \%$ aqueous ethanol $(5 \times 20$ $\mathrm{mL})$ and water $(5 \times 20 \mathrm{~mL})$. Blank polymers were not hydrolyzed. Instead, they were washed with $0.5 \mathrm{M} \mathrm{NaOH}$ in $50 \%$ ethanol-water $(5 \times 20 \mathrm{~mL})$ and water $(10 \times 20 \mathrm{~mL})$. Then all polymers were washed with dilute $\mathrm{HCl}$ by slowly passing $150 \mathrm{~mL}$ of $0.5 \%$ acid solution through the polymers for $30 \mathrm{~min}$ to recover the free carboxylic acid groups. Polymers were then washed with copious amounts of distilled water until the $\mathrm{pH}$ of the eluent was neutral, followed by methanol $(3 \times 20 \mathrm{~mL})$. Fine particles were removed by washing the polymers with methanol on a $45 \mu \mathrm{m}$ sieve. Polymers were stored in methanol. Suspensions in methanol were used for packing the HPLC columns. Blank polymer P4 was prepared by the same procedure in the absence of the template phenol derivative, i.e. $3 \mathrm{~g}$ of divinylbenzene was used for the polymerization. Polymer P5, containing itaconic acid instead of one of the hydroxyphenol monomers, was prepared as a further control in the same way. The amount of acid in the polymerization mixtures was adjusted so that carboxylic acid content in P5 was close to that in the imprinted polymers P1-P3.

\section{Polymer Titration}

Potentiometric titration, which was used to determine the amount of carboxylic groups in the polymers, was done as described previously [15]. Carefully weighed dried polymer samples (400-500 mg) were each suspended in $20 \mathrm{~mL}$ of water containing $10 \%$ methanol (for better wetting of hydrophobic polymers). The suspension was magnetically stirred continuously throughout. Aqueous $\mathrm{NaOH}$ solution (1 $\mathrm{N})$ was added until the $\mathrm{pH}$ of the suspension reached 12 and the mixture was left to equilibrate for $30 \mathrm{~min}$. The titrant solution $(0.996 \mathrm{~N} \mathrm{HCl})$ was then added in $20 \mu$ aliquots and the $\mathrm{pH}$ recorded after stabilization (ca. 10 min after each addition). The progress of the titration was monitored with a Hanna $8519 \mathrm{pH}$-meter (Hanna Instruments) equipped with a glass electrode (Fisher). The titration was continued until the $\mathrm{pH}$ reached 2.3-2.4. The $\mathrm{pH}$ values were then plotted against titrant volume. The content of $-\mathrm{CO}_{2} \mathrm{H}$ groups was calculated 
from the amount of $\mathrm{HCl}$ necessary to neutralize the polymerbound sodium carboxylate groups. The $\mathrm{pK}_{\mathrm{a}}$ value was derived from the plot as the $\mathrm{pH}$ corresponding to $50 \%$ ionisation.

\section{HPLC Analysis}

HPLC was performed on a system consisting of ConstaMetric 3200 solvent delivery system (LDC Analytical, UK), Waters 717 Plus autosampler and LambdaMax 481 LC spectrophotometric detector (Waters, UK). HPLC columns $(4.6 \times 100 \mathrm{~mm})$ were packed with polymer $(1 \mathrm{~g})$ in methanol. For HPLC analysis, $1 \mathrm{mg} \mathrm{mL}^{-1}$ sample solutions in acetonitrile, chloroform or $2 \% \mathrm{MeOH} / \mathrm{CHCl}_{3}$ were prepared, $20 \mu \mathrm{L}$ was used for injection. HPLC was run at a flow rate of $1 \mathrm{~mL} \mathrm{~min}{ }^{-1}$ (isocratic elution, $10 \mathrm{~min}$ ) with detection at $254 \mathrm{~nm}$. Eluent composition was optimized for each polymer and set of compounds to be analyzed. Reported chromatographic data represent the results of at least two concordant experiments. The standard deviation in the experiments was below $5 \%$. Capacity factors $k^{\prime}$ were determined from the equation $k^{\prime}=\left(t-t_{0}\right) / t_{0}$, where $t$ is the retention time of the given species and $t_{0}$ is the retention time of the void standard (acetone).

\section{Computational Analysis of the Binding Sites}

Molecular modelling was performed on a Silicon Graphics Octane workstation running IRIX 6.5 operating system. The workstation was configured with two $195 \mathrm{MHz}$ reduced instruction set processors, $712 \mathrm{MB}$ memory and a 12 GB fixed drive. This system was used to execute the software package SYBYL 6.7 (Tripos Inc., USA). Analysis of the distances between functional groups in the corresponding molecular models was performed using the FlexiDock algorithm, which is an integral component of the SYBYL molecular modelling environment, and is provided as a part of its Biopolymer module. At the beginning of the experiment, molecular models of diazine heterocycles, hydroxypyridines and hydroxyphenols, diacrylate monomers and acrylic acid were created. Next the SYBYL's docking function was used to position two molecules of acrylic acid in the most energetically favourable orientation around the substrate molecules. The distances between carboxylic functionalities were calculated and compared with the distances between carboxyl groups in the diacrylate monomers.

\section{Swelling Analysis}

Swelling experiments were performed as described previously [16]. Three hundred milligrams of the polymer particles with mesh size $38-67 \mu \mathrm{m}$ were packed in $1-\mathrm{mL}$ solid-phase extraction cartridges (Supelco, UK). Cartridges were filled with $1 \mathrm{~mL}$ of chloroform. After 6 hours equilibration at $20{ }^{\circ} \mathrm{C}$, excess solvent was removed from the polymer by applying reduced pressure for 1 minute and the weight of the swollen polymer was measured. The swelling ratio $(S r)$ of the polymers was calculated from the following equation $S r=\left(m_{s}-m_{o}\right) / m_{o}$, where $m_{s}$ is the mass of the swollen polymer and $m_{o}$ is the mass of dry polymer.

\section{RESULTS AND DISCUSSION}

The general principles of molecular imprinting have been used in this work; however the intention was not to create recognition sites specific to the species used as templates, but rather to prepare a series of chemically identical polymers which differ in the orientation of functional groups around a similarly sized cavity. This has been achieved using a covalent template approach with the aim of preparing materials with general specificity to compounds, not necessarily structurally related to the template, which possess functionality complementary to that of the polymer sites. The high level of cross-linker used in the formulations helps to preserve the orientation and distances of the groups within the polymers by formation of relatively rigid theedimensional networks. In the first example of this approach, bis-esters of the regioisomers of hydroxyphenol were used to position carboxylic acid groups at variable distances and orientations around the template cavities.

Acrylate and methacrylate esters of phenols can be prepared by acylation with the corresponding acyl chloride in the presence of hindered pyridine bases such as 2,6lutidine, which prevent polymerization occurring during formation of the esters (this is especially important for phenols bearing electron-withdrawing substituents) $[17,18]$. Acylation reactions can be also performed in the presence of triethylamine (TEA) [8,19]. A modification of the latter procedure was used to prepare polymerizable derivatives of the hydroxyphenols, namely catechol, resorcinol and hydroquinone diacrylates (Fig. 1). The diacrylate monomers were obtained in good yield $(61-66 \%)$ following purification by column chromatography and the structures were confirmed by mass spectrometry.

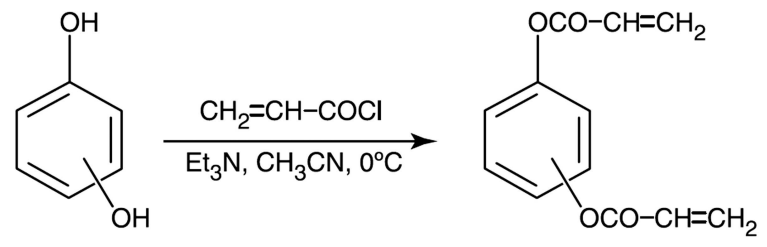

Fig. (1). Synthesis of diacrylate monomers.

A set of cross-linked polymers, P1-P3, was synthesized using three phenol diacrylate monomers as templates (Fig. 2). Thermal free radical polymerization was carried out in $\mathrm{DMF}$ at $80{ }^{\circ} \mathrm{C}$ in the presence of 1,1'-azobis(cyclohexanecarbonitrile) as initiator. The cross-linker (divinylbenzene) and diacrylate monomers were present at a ratio of 9:1 (w/w) in the polymerization mixtures (Table $\mathbf{1}$ ).

Template removal was achieved by hydrolysis with aqueous $\mathrm{NaOH}$ to cleave the phenolic residues from the polymers with the formation of free carboxylic groups within the template cavities. Alkaline solutions of phenols are prone to atmospheric oxidation leading to the formation of high molecular weight products. Darkening of the reaction mixtures was observed, so in order to minimize the likelihood of phenol oxidation products being adsorbed on the polymer surfaces, the $\mathrm{NaOH}$ solution was changed several times throughout the course of the hydrolysis reactions. The end-point of the ester hydrolysis was judged to have been reached when a fresh portion of the alkaline solution added to the polymer did not darken, indicating that no further free phenol had been formed. Aryl esters are known to be readily hydrolyzed in base, this was confirmed for the diacrylate esters by model experiments using similar reaction conditions to those employed with the polymers 


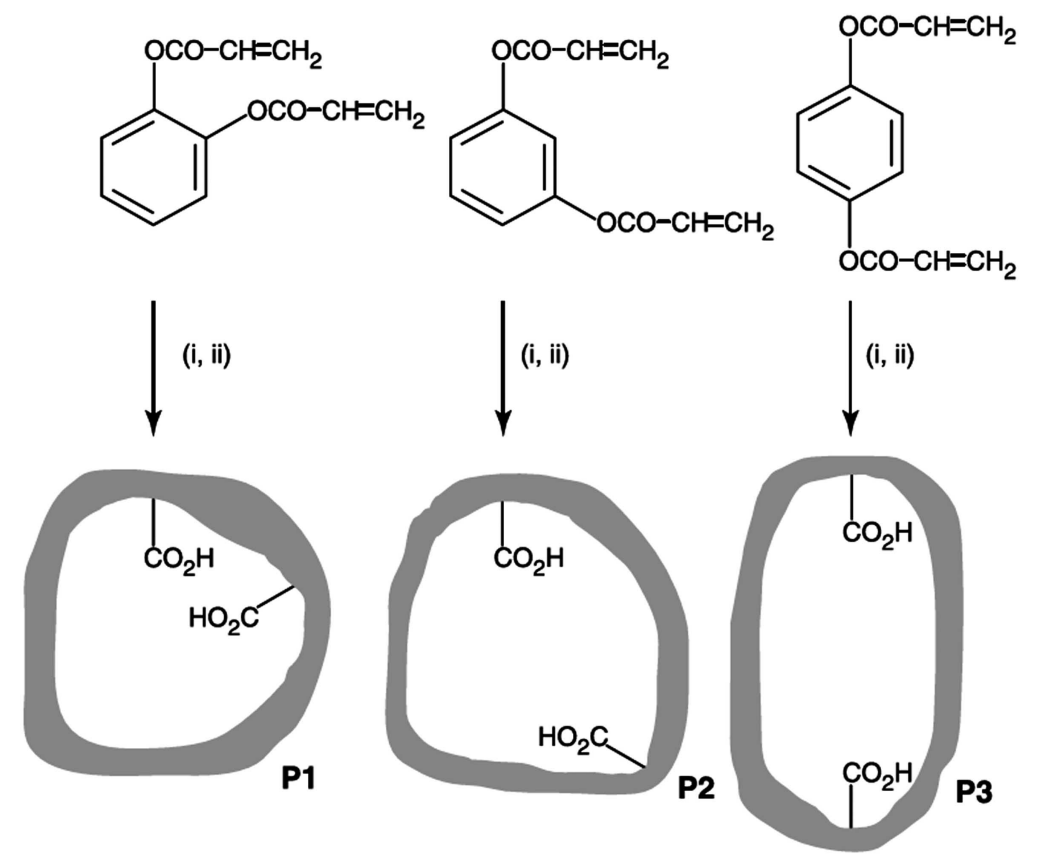

Fig. (2). Preparation of polymers P1-P3 from polymerizable esters: (i) - polymerization, (ii) - alkaline hydrolysis. Highlighted are the schematic representations of binding sites created in the polymers.

Table 1. Composition of Polymerization Mixtures Used for the Preparation of Polymers

\begin{tabular}{|c|c|c|c|c|c|}
\hline Polymer & Functional Monomer, $\mathbf{m g}$ & Cross-Linker ${ }^{\mathbf{a}}, \mathbf{g}$ & Initiator ${ }^{\mathbf{b}}, \mathbf{m g}$ & DMF, $\mathbf{g}$ & Swelling Ratio, ${ }^{\boldsymbol{c}} \boldsymbol{S r}$ \\
\hline \hline P1 & Catechol diacrylate, 300 & 2.7 & 30 & 3.0 & 1.20 \\
\hline P2 & Resorcinol diacrylate, 300 & 2.7 & 30 & 3.0 & 1.19 \\
\hline P3 & Hydroquinone diacrylate, 300 & 2.7 & 30 & 3.0 & 1.15 \\
\hline P4 (blank) & - & $3 \mathrm{~g}$ & 30 & 3.0 & n. d. \\
\hline P5 & Itaconic acid, 114 & 2.8 & 30 & n. d. \\
\hline
\end{tabular}

${ }^{\mathrm{a}}$ Divinylbenzene; ${ }^{\mathrm{b}} 1,1^{\prime}$-azobis(cyclohexanecarbonitrile); ${ }^{\mathrm{c}} \mathrm{Sr}=(\mathrm{ms}-\mathrm{mo}) / \mathrm{mo}$; n. d.- not determined.

(0.75 $\mathrm{M} \mathrm{NaOH}$ in $50 \%$ aqueous methanol, $60{ }^{\circ} \mathrm{C}$ ). Under these conditions the most labile resorcinol derivative was completely cleaved in $30 \mathrm{~min}$, whereas catechol and hydroquinone diacrylates were hydrolyzed in $2 \mathrm{~h}$ at ambient temperature.

Titration of the polymers showed that very similar amounts of carboxyl groups were formed when polymers, subjected to hydrolysis for 10 and $24 \mathrm{~h}$, were compared. For example, hydrolysis of $\mathbf{P 2}$ for $10 \mathrm{~h}$ gave $0.15 \mathrm{mmol} \mathrm{g}^{-1}$ of titrable $\mathrm{CO}_{2} \mathrm{H}$ groups, whereas exhaustive hydrolysis of the same polymer for $24 \mathrm{~h}$ showed only a marginally higher carboxylic acid group content at $0.17 \mathrm{mmol} \mathrm{g}$. Prolonged alkaline treatment therefore gave very little improvement in the carboxylic groups content of the polymers and consequently hydrolysis for 8-10 h appeared to be sufficient for the almost complete hydrolysis of the accessible phenol ester residues. All hydrolyzed polymers were therefore compared on this basis and the results are reported on Table 2.

Polymers P1-P3 were prepared using $100 \mathrm{mg}$ (0.46 mmol) of hydroxyphenol diacrylate monomer per $1 \mathrm{~g}$ of monomer mixture, thus the estimated maximum concentration of carboxylic groups in the dry polymers after
Table 2. Carboxylic Group Content and Acidic Properties of Obtained Polymers and Monomers

\begin{tabular}{|c|c|c|}
\hline Polymer & {$[\mathbf{C O O H}], \mathbf{~ m m o l ~ g}^{-1}$} & $\mathbf{p K}_{\mathbf{a}}$ \\
\hline \hline P1 & 0.15 & 5.1 \\
\hline P2 & 0.15 & 5.1 \\
\hline P3 & 0.17 & 5.0 \\
\hline P4 & 0 & - \\
\hline P5 & 0.16 & 4.7 \\
\hline Itaconic Acid & - & 3.85 and 5.45 [20] \\
\hline
\end{tabular}

complete hydrolysis would be $0.92 \mathrm{mmol}^{-1}$. The experimentally determined figures were in the range of 0.15 $0.17 \mathrm{mmol} \mathrm{g}^{-1}$, meaning that only $16-18 \%$ of the phenolic ester residues were accessible for hydrolytic cleavage. Other possible explanations for the low titration results could be that some of the carboxylic groups formed were inaccessible to the base used for titration, alternatively, less than the expected amount of diacrylate monomer was incorporated into the cross-linked polymer. Swelling experiments 
(Table 1) demonstrated that the polymers P1-P3 only swell on average $15-20 \%$. This swelling might not be sufficient to expose all functional groups in the cross-linked polymeric domains for the hydrolysis and may also effect the titration.

The selectivity of the hydrolyzed polymers for phenol templates and structurally related compounds was studied using sets of substrates that contain pairs of functional groups in the ortho-, meta- and para-orientations. These included the starting phenols (catechol, resorcinol, hydroquinone), the diazine heterocycles (pyridazine, pyrimidine, pyrazine), and 2-, 3- and 4-hydroxypyridine. The functional groups in the selected structures $\left(-\mathrm{OH},-\mathrm{NH}_{2}\right.$ and aromatic nitrogen) are able to interact with $\mathrm{CO}_{2} \mathrm{H}$ groups through electrostatic interactions. The retention properties of the samples were analyzed by isocratic mode HPLC on columns packed with each of the polymers. The experimental data for imprinted polymers are presented in the Table $\mathbf{3}$.

It should be noted that the chromatographic properties of three sets of compounds, which were analyzed (hydroxyphenols, dinitrogen heterocycles and hydroxypyridines), differ quite markedly from each other, so it was not possible to perform chromatography efficiently using the same solvent for all compound types. As a result it was necessary to optimize the eluent composition for each class of compound. The retention times for the different compounds tested will depend on their intrinsic properties; such as pKa, the ability to form hydrogen bonds as well as tautomeric effects (2- and 4-hydroxypyridine exist mainly in the 2- and 4-pyridone form in apolar solvents). To predict their retention on each of the polymers would be very challenging and it was not our intention to do so. The focus of this research was to find differences in the binding properties of the imprinted polymers P1-P3, which are in essence chemically very similar materials. If it was not for the structural differences within imprinted cavities, these materials would have very similar capacity factors with any of the test compounds. The results of HPLC experiments clearly indicate that the nature of the template had an affect on the affinity profile of the synthesized polymers. For example, ortho-and para-hydroxyphenols (catechol and hyroquinone, respectively) had greatest affinity for polymers $\mathbf{P 1}$ and P3, respectively, prepared from the diacrylate derivatives of these phenols. Pyridazine (ortho-orientation of two nitrogen atoms) had the highest affinity to the polymer P1 prepared from the catechol derivative (orthosubstitution), and pyrazine (para-diazine) had the strongest binding to the polymer $\mathbf{P 3}$ prepared using the parasubstituted monomer, derived from hydroquinone. Orthoand para-isomeric hydroxypyridines also showed the strongest retention on polymers $\mathbf{P 1}$ and $\mathbf{P 3}$, respectively. In general ortho- and para-substrates were better recognized by polymers where the carboxylic acid groups were oriented accordingly. However, meta-substituted substrates (resorcinol, pyrimidine and 3-hydroxypyridine) were not retained most efficiently by the polymer (P2) with the metaorientation of $-\mathrm{CO}_{2} \mathrm{H}$ groups.

The reason in the latter case could be that the distance between carboxylic functions and their orientation in the templated sites of $\mathbf{P} \mathbf{2}$ are not optimal for binding to the polar groups in substrates with the meta-orientation of functional groups. In order investigate this phenomenon, an analysis of the distances between interacting groups was performed using a molecular modelling approach.

Molecular modelling of the binding sites in the polymers P1-P3 was performed using the SYBYL software package. The assumption was made that the distance between carboxylic functions in the polymeric cavities is identical to that in the constituent diacrylate monomers. The optimum distance between $-\mathrm{CO}_{2} \mathrm{H}$ groups in the complexes of acrylic acid with substrates are presented in Table 4.

The possible orientations of acrylic acid in the complexes with diazine heterocycles is presented in Fig. (3).

In general, the results suggest that ortho- and parasubstrates should be better recognized by the corresponding ortho- and para-polymers (P1 and P3, respectively). For example according to the obtained data the best polymer for the recognition of pyridazine should be the one prepared using the catechol derivative (P1). Similarly, pyrazine should have the strongest binding to the polymer prepared using hydroquinone (P3). These results are in accordance with experimental data from the chromatographic analysis of polymer performance. In the case of pyrimidine, in which

Table 3. Capacity Factors $\left(k^{\prime}\right)$ of the Tested Compounds Measured for Columns Packed with Imprinted Polymers

\begin{tabular}{|c|c|c|c|c|c|}
\hline Substrate & Solvent & \multicolumn{4}{|c|}{$k^{\prime}$ of the Polymer } \\
\hline Resorcinol (meta-) & $\mathrm{CHCl}_{3}$ & 4.30 & 4.10 & 3.98 & 0.47 \\
\hline Hydroquinone (para-) & $\mathrm{CHCl}_{3}$ & 2.55 & 2.70 & 2.90 & 0.34 \\
\hline Pyrimidine (meta-) & $\mathrm{CHCl}_{3}, 20 \%$ hexane & 1.46 & 1.67 & 2.37 & n. d. \\
\hline Pyrazine (para-) & $\mathrm{CHCl}_{3}, 20 \%$ hexane & 0.84 & 0.77 & 1.66 & n. d. \\
\hline 2-Hydroxy-pyridine (ortho-) & $\mathrm{CHCl}_{3}, 3 \% \mathrm{MeOH}$ & 2.50 & 1.94 & 2.10 & n. d. \\
\hline 3-Hydroxy-pyridine (meta-) & $\mathrm{CHCl}_{3}, 3 \% \mathrm{MeOH}$ & 0.99 & 0.85 & 1.29 & n. d. \\
\hline
\end{tabular}


Table 4. Calculated Distance Between Carboxylic Groups in the Polymeric Binding Site and in the Complexes Between Acrylic Acid and Heterocycles (as Shown in Fig. 1)

\begin{tabular}{|c|c|c|c|c|}
\hline \multirow{2}{*}{ Derivative } & \multirow{2}{*}{$\begin{array}{c}\text { Calculated Distance Between } \\
\text { Carboxylic Groups in } \\
\text { Binding Site, } \AA\end{array}$} & \multicolumn{3}{|c|}{ Optimal Distance Between Carboxylic Groups in Complexes of Acrylic Acid with Analyte, $\AA$} \\
\cline { 3 - 5 } & 2.8 & Hydroxyphenols & Hydroxypyridines & \multirow{2}{*}{ Diazines } \\
\hline \hline Ortho- & 4.8 & $2.9-5.0$ & $1.5-2.7$ & $1.6-3.0$ \\
\hline Meta- & 5.5 & $3.5-4.8$ & $4.4-5.8$ & $3.1-5.3$ \\
\hline Para- & $6.6-8.3$ & $4.2-7.6$ & $5.8-8.0$ \\
\hline
\end{tabular}

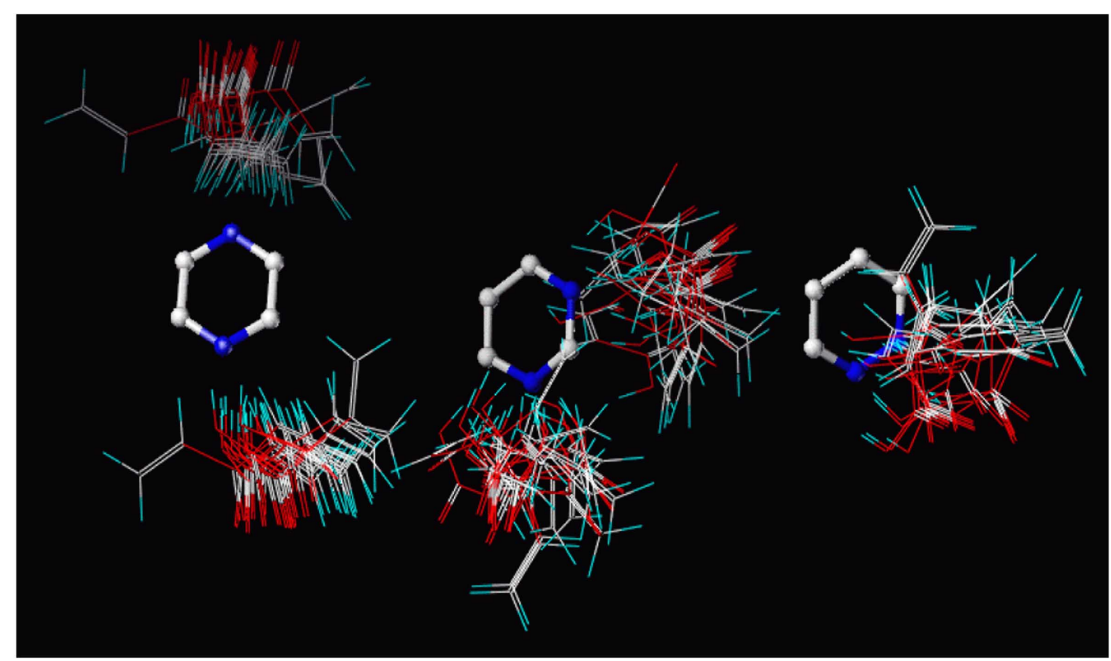

Fig. (3). Possible orientations of acrylic acid in the complexes with diazine heterocycles.

the polar nitrogen moieties are in the meta-orientation, the prognosis is difficult to make since all three polymers should be capable of binding this heterocycle. Similar results were obtained for hydroxyphenols and hydroxypyridines. Again, ortho- and para-substituted substrates are better suited for the interaction with ortho- and para-polymers as the optimal interaction distances are close to those calculated between $\mathrm{CO}_{2} \mathrm{H}$ groups in the binding sites of the corresponding polymers. The optimal distances for meta-substrates, as a rule, more closely correspond to the distances in orthoand/or para oriented binding sites (Table 4). Thus, the synthesized materials have selectivity not only for the phenolic templates, but also for a group of compounds with suitable orientation of polar functionalities, such as aromatic nitrogen, amino or hydroxyl groups.

The ability of imprinted cavities to accommodate different molecules is facilitated by polymer swelling (15$20 \%$ ). It would be important in the future to investigate to what degree swelling determines the functional properties and practical performance of the synthesized materials.

Blank polymer $\mathbf{P 4}$ produced from divinylbenzene in the absence of any phenol diacrylate monomer and treated in the same way as other polymers demonstrated very low retention time for all compounds tested. Similarly non-hydrolyzed polymers did not show any noticeable binding to these analytes. It is clear that the presence of carboxylic groups in the polymers is necessary for interaction with analyzed compounds.

Another control, non-imprinted polymer, P5 was prepared using itaconic acid as functional monomer. The $\mathrm{CO}_{2} \mathrm{H}$ group content in this polymer $\left(0.18 \mathrm{mmol} \mathrm{g}^{-1}\right)$ was close to the concentration seen in the templated polymers
P1-P3 (0.15-0.17 mmol $\left.\mathrm{g}^{-1}\right)$. Itaconic acid contains two carboxylic acid groups in close proximity that in principle can cooperate in their interaction with the compounds analyzed in a similar way to some of the templated polymers P1-P3. It was interesting to see if any evidence existed for co-operative effects in the binding between heterocycles and the itaconic acid polymer, as compared with polymers P1P3. Indeed, itaconic acid-containing polymer P5 retained all test compounds more efficiently than polymers P1-P3 (Table 5).

This may not be surprising however, since itaconic acid and itaconic acid-based polymers are more acidic than acrylic acid and its polymers (Table 2 ). As a consequence the retention of heterocycles on the itaconic acid-based polymer was in direct correlation with the relative basicity of the test compounds: pyridazine $>$ pyrimidine $>$ pyrazine (Table 5). In contrast to P5 however, the imprinted polymers P1-P3 demonstrated orientation-dependent co-operative effects in their binding which can be seen from the obtained results (Tables 3 and $\mathbf{5}$ ).

In future work we intend to modify polymeric recognition sites by creation of a range of functionalities in polymers suited for the recognition of different substrate molecules. For example, combinations of hydroxyl and amino groups or carboxyl and hydroxyl groups at various, relatively fixed distances can be introduced into polymers by a variety of synthetic routes. Such polymers could discriminate between substrates with different orientation of polar substituents. Potentially limited set of polymers with fixed positions of two functional groups in a binding cavity would facilitate the separation of a broad range of 
Table 5. Capacity Factors ( $\left.k^{\prime}\right)$ of the Tested Compounds on Imprinted (P1-P3) and Non-Imprinted (P5) Polymers in Chloroform Containing $20 \%$ Hexane

\begin{tabular}{|c|c|c|c|}
\hline \multirow{2}{*}{ Polymer } & \multicolumn{3}{|c|}{ k, } \\
\cline { 2 - 4 } & Pyridazine (ortho-) & Pyrimidine (meta-) & Pyrazine (para-) \\
\hline \hline P1 (ortho-) & 3.47 & 1.46 & 0.84 \\
\hline P2 (meta-) & 1.75 & 1.67 & 0.77 \\
\hline P3 (para-) & 2.03 & 2.37 & 1.66 \\
\hline P5 (IA) & 10.30 & 9 & 5.74 \\
\hline$p K_{a}[21,22]$ & $2.10-2.24$ & $1.10-1.30$ & $0.37-0.65$ \\
\hline
\end{tabular}

compounds. Such separation would facilitate the detection and quantification of the separated compounds, even when they are contained in challenging sample matrix. This type of polymeric adsorbent has the potential to become a generic tool for modern separation science, filling a niche between non-specific adsorbents such as e.g. ion exchangers and highly specialized imprinted polymers or immunoadsorbents.

\section{ACKNOWLEDGEMENT}

Declared none.

\section{CONFLICTS OF INTEREST}

Declared none.

\section{REFERENCES}

Alexander, C.; Andersson, H.S.; Andersson, L.I.; Ansell, R.J.; Kirsch, N.; Nicholls, I.A.; O'Mahony, J.; Whitcombe, M.J. Molecular imprinting science and technology: a survey of the literature for the years up to and including 2003. J. Mol. Recognit., 2006, 19, 106-180.

[2] Lasakova, M.; Jandera, P. Molecularly imprinted polymers and their application in solid phase extraction. J. Sep. Sci., 2009, 32, 799-812.

[3] Whitcombe, M.J.; Chianella, I.; Larcombe, L.; Piletsky, S.A.; Noble, J.; Porter, R.; Horgan, A. The rational development of molecularly imprinted polymer-based sensors for protein detection, Chem. Soc. Rev., 2011, 40, 1547-1571.

[4] Xu, Z.X.; Gao, H.J.; Zhang, L.M.; Chen, X.Q.; Qiao, X.G. The biomimetic immunoassay based on molecularly imprinted polymers: A comprehensive review of recent progress and future prospects. J. Food Sci., 2011, 76, R69-R75.

[5] Carboni, D.; Flavin, K.; Servant, A.; Gouverneur, V.; Resmini, M. The first example of molecularly imprinted nanogels with aldolase type I activity. Chem. Eur. J., 2008, 14, 7059-7065.

[6] Mayes, A.G.; Whitcombe, M.J. Synthetic strategies for the generation of molecularly imprinted organic polymers. Adv. Drug Deliv. Rev., 2005, 57, 1742-1748.

[7] Chianella, I.; Lotierzo, M.; Piletsky, S.A.; Tothill, I. E.; Chen, B.; Karim, K.; Tuner, A.P.F. Rational design of a polymer specific for microcystin-LR using a computational approach. Anal. Chem., 2002, 74, 1288-1293.

[8] Ye, L.; Cormack, P.A.G.; Mosbach, K. Molecular imprinting on microgel spheres. Anal. Chim. Acta, 2001, 435, 187-196.

[9] Caro, E.; Masqué, N.; Marcé, R.M.; Borrull, F.; Cormack, P.A.G.; Sherrington, D.C. Non-covalent and semi-covalent molecularly imprinted polymers for selective on-line solid-phase extraction of 4-nitrophenol from water samples. J. Chromatogr. A, 2002, 963, 169-178.
[10] Caro, E.; Marcé, R.M.; Cormack, P.A.G.; Sherrington, D.C.; Borrull, F. On-line solid-phase extraction with molecularly imprinted polymers to selectively extract substituted 4chlorophenols and 4-nitrophenol from water. J. Chromatogr. A, 2003, 995, 233-238.

[11] Ikegami, T.; Mukawa, T.; Nariai, H.; Takeuchi, T. Bisphenol Arecognition polymers prepared by covalent molecular imprinting. Anal. Chim. Acta, 2004, 504, 131-135.

[12] Ikegami, T.; Lee, W.S.; Nariai, H.; Takeuchi, T. Synthetic polymers adsorbing bisphenol $\mathrm{A}$ and its analogues prepared by covalent molecular imprinting using bishopenol A dimethacrylate as a template molecule. Anal. Bioanal. Chem., 2004, 378 , 18981902.

[13] Kubo, T.; Tanaka, N.; Hosoya, K. Target-selective ion-exchange media for highly hydrophilic compounds: a possible solution by use fo "interval immobilization technique". Anal. Bioanal. Chem., 2004, 378, 84-88.

[14] Kubo, T.; Hosoya, K.; Watabe, Y.; Tanaka, N.; Takagi, H.; Sano, $\mathrm{T}$.; Kaya, K. Interval immobilization technique for recognition towards a highly hydrophilic cyanobacterium toxin. J. Chromatogr. $B$, 2004, 806, 229-235.

[15] Piletska, E.V.; Guerreiro, A.R.; Romero-Guerra, M.; Chianella, I.; Turner, A.P.F.; Piletsky, S.A. Design of molecular imprinted polymers compatible with aqueous environment. Anal. Chim. Acta, 2008, 607, 54-60.

[16] Piletsky, S.A.; Mijangos, I.; Guerreiro, A.; Piletska, E.V.; Chianella, I.; Karim, K.; Turner, A.P.F. Polymer Cookery III: Influence of Polymerization Time and Different Initiation Conditions on Performance of Molecularly Imprinted Polymers. Macromolecules, 2005, 38, 1410-1414.

[17] Blazejewski, J.C.; Hofstraat, J.W.; Lequesne, C.; Wakselman, C.; Wiersum, U.E. Formation of monomeric halogenoaryl acrylates in the presence of hindered pyridine bases. J. Fluor. Chem., 1998, 91, 175-177.

[18] Blazejewski, J.C.; Hofstraat, J.W.; Lequesne, C.; Wakselman, C.; Wiersum, U.E. Halogenoaryl acrylates: preparation, polymerization and optical properties. J. Fluorine Chem., 1999, 97, 191-198.

[19] Thamizharasi, S.; Gnanasundaram, P.; Reddy, B.S.R. Copolymerization of 4-acetyl phenylacrylate with methyl methacrylate and butyl methacrylate: Synthesis, characterization, and reactivity ratios. Eur. Polym. J., 1997, 33, 1487-1494.

[20] Merkli, A.; Heller, J.; Tabatabay, C.; Gurny, R. The use if acidic and basic excipients in the release of 5-fluorouracil and mitomycin C from a semi-solid bioerodible poly (ortho ester). J. Control Release, 1995, 33, 415-421.

[21] Brown, H.C.; McDaniel, D.H.; Häfliger, O., The Determination of Organic Structures by Physical Methods; Braude E.A.; Nachod F.C. (Eds); Academic Press, New York, 1955.

[22] Albert A.; Serjeant E.P The Determination of Ionization Constants. A Laboratory Manual; Chapman and Hall, London, 1984. 